\title{
Estimation of Spurious Radiation from Microstrip Etches Using Closed-Form Green's Functions
}

\author{
M. Irsadi Aksun and Raj Mittra, Fellow, IEEE
}

\begin{abstract}
The problem of spurious radiation from electronic packages is considered in this paper by investigating the power radiated from microstrip etches that are excited by arbitrarilylocated current sources, and terminated by complex loads at both ends. The first step in the procedure is to compute the current distribution on the microstrip line by using the method of moments (MoM). Two novel contributions of this paper are: (i) employing the recently-derived closed-form Green's functions in the spatial domain that permit an efficient computation of the elements of the MoM matrix; (ii) incorporating complex load terminations in a convenient manner with virtually no increase in the computation time. The computed current distribution is subsequently used to calculate the spurious radiated power and the result is compared with that derived by using an approximate, transmission line analysis.
\end{abstract}

\section{INTRODUCTION}

$\mathrm{O}^{\mathrm{N}}$ NE OF THE most commonly-used numerical techniques for solving electromagnetic problems is the method of moments (MoM), which is based upon the transformation of an operator equation into a matrix equation [1]. Although the MoM is preferred over differential equation methods for the microstrip circuits and radiation problems because it is relatively efficient in terms of computation time, MoM is still quite time-consuming to use owing to the oscillatory nature and slow convergence of the integrals involved. One approach to alleviating the above difficulties is to employ closed-form Green's functions in the spatial domain, that can speed up the computation of the MoM matrix elements for planar microstrip structures by several orders of magnitude as compared to the Sommerfeld integral or spectral domain method. Closed-form Green's functions in the spatial domain have been derived recently for microstrip geometries on a thick substrate by Chow et al. [2]-[3], and extended to general microstrip geometries with a substrate and a superstrate of arbitrary thickness by Aksun and Mittra [4]. Once the improper and infinite range integrals for the Green's functions have been expressed in closedforms, the remaining integrals need be computed only over finite supports associated with the basis and testing functions. In this paper, we present the application of the

Manuscript received November 14, 1991; revised March 30, 1992.

M. I. Aksun was with the Department of Electrical and Computer Engineering, University of Illinois at Urbana-Champaign, Urbana, IL 61801 He is presently with the Department of Electrical and Electronics Engineering, Bilkent University, 06533, Ankara, Turkey.

R. Mittra is with the Department of Electrical and Computer Engineering, University of Illinois, at Urbana-Champaign, Urbana, IL 61801.

IEEE Log Number 9202899. closed-form Green's functions to the problem of spurious radiation calculation from an interconnect that is typically modeled as a microstrip line fed by a current source and terminated by complex loads at both ends. Our objective is to compute the current distribution on the line and the level of spurious radiation, as functions of the length of the line and the load impedances, under the assumption that both the location of the current source and the complex load impedances terminating the line are arbitrary.

The current distribution on a microstrip line is calculated in Section II of this paper by using the Galerkin's method in the spatial domain that incorporates the closedform Green's functions in the MoM matrix computation. We begin by presenting a brief description of the application of the MoM and the derivation of a set of linear equations for computing the current distribution on a microstrip line fed by a localized current source. Since the current density on the line is expanded in terms of basis functions in the context of MoM, the choice of these functions is important from the point of view of the convergence of the integrals involved [5], and we include a brief discussion of this issue in this section. Next, we compute the current distributions for various lengths and load impedances and compare these results with those calculated by using an approximate transmission line (TL) model for the problem.

In Section III, the level of spurious radiation, which is defined as the radiated power crossing the plane parallel to the plane of the microstrip line, is calculated by making use of the current distribution obtained in the previous section. The results for the radiated power for some representative termination impedances are given as functions of the line length of the microstrip etch.

\section{Current Distribution on a Microstrip Line Terminated By COMPlex LOADS}

Fig. 1 shows the geometry of a microstrip line fed by an arbitrarily-located current source and terminated by complex impedances at both ends. The substrate is assumed to be infinitely wide in the $x$-and $y$-directions with a thickness $d_{1}$ and a relative permittivity $\epsilon_{r 1}$. The superstrate above is air for this example.

\section{A. Formulation of the Problem}

The electric field along the line can be expressed in terms of the surface current density $J$ and the vector and scalar Green's functions, $G_{x x}^{A}$ and $G_{q}$, respectively, as fol- 


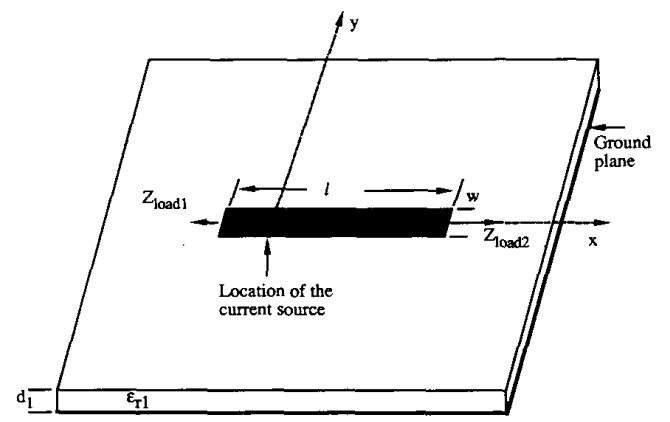

Fig. 1. Geometry of a microstrip line terminated by complex loads

lows:

$$
E_{x}=-j \omega G_{x x}^{A} * J_{x}+\frac{1}{j \omega} \frac{\partial}{\partial x}\left(G_{q} * \nabla \cdot J\right),
$$

where * implies convolution. The derivation of the closed form expressions for the Green's functions have been described in detail in [4] and will be omitted here. In the above equation we have assumed that the $y$-component of the current density is negligible, which is justified because the width of the microstrip line $w$ is much smaller than the wavelength in the dielectric medium. Next, we express the $x$-directed current density in terms of the basis functions as

$$
J_{x}(x, y)=\sum_{n} I_{n} J_{x n}(x, y)+J_{s}(x, y)
$$

where $I_{n}$ is the unknown coefficient of the basis function and $J_{s}$ is the basis function associated with the current source. The choices of these basis functions will be detailed in the next section from the convergence point of view. Upon substituting (2) into (1), and testing the resulting equations with the basis functions $J_{x m}$, i.e., following the Galerkin's procedure with a suitable definition of inner product, we obtain the following algebraic equation for coefficients $I_{n}$ for each $m$ :

$$
\begin{gathered}
\left.\sum_{n} I_{n}\left[\left\langle J_{x m}, G_{x x}^{A} * J_{x n}\right\rangle+\frac{1}{\omega^{2}} \quad J_{x m}, \frac{\partial}{\partial x}\left(G_{q} * \frac{\partial}{\partial x} J_{x n}\right)\right\rangle\right] \\
=-\left\langle J_{x m}, G_{x x}^{A} * J_{s}\right\rangle-\frac{1}{\omega^{2}} \cdot J_{x m}, \frac{\partial}{\partial x}\left(G_{q} * \frac{\partial}{\partial x} J_{s}\right)
\end{gathered}
$$

The number of equations, i.e., $m$, must be commensurate with the number of unknowns $n$, or additional conditions must be imposed in order that the resultant matrix is square and the solution for the coefficients $I_{n}$ is unique.

\section{B. Choices of Basis Functions}

It is well-known that the choice of the basis and testing functions plays an important role in determining the rate of convergence of the integrals associated with the moment method matrix (3). An improper choice can lead to non-convergent integrals [5] and, consequently, erroneous results [6]. After a thorough examination of the convergence of the integrals involved in the MoM matrix, the basis and testing functions must satisfy the following criteria [5]: (i) In the direction of the polarization of the current, the sum of the order of the differentiability of the basis and testing functions must be equal to or greater than one; (ii) in the orthogonal direction of the polarization of the current, any piecewise continuous function or even functions with singularities of the order of less than one are admissible.

In view of the above criteria, the basis functions, apart from those that represent the source and load currents, are chosen to be rooftops, which are triangular functions in the longitudinal direction, uniform in the transverse direction, and are defined mathematically as

$$
J_{x m}(x, y)= \begin{cases}\frac{1}{w}\left[\begin{array}{l}
\left.1-\frac{\left|x-m h_{x}\right|}{h_{x}}\right] \\
(m-1) h_{x} \leq x \leq(m+1) h_{x}
\end{array}\right. & |y| \leq w / 2 \\
0 & \text { elsewhere }\end{cases}
$$

where $2 h_{x}$ is the support of the basis functions (see Fig. 2(a)).

The source and load contributions to the current density on the microstrip line are taken into account by employing suitable basis functions for them and relating them to the other equations. The basis functions for the current distribution associated with the source and loads are given by

$$
\begin{gathered}
J_{s}(x, y)=\left\{\begin{array}{cl}
\frac{-1}{w}\left(1+\frac{x}{h_{x}}\right) & -h_{x} \leq x \leq 0,|y| \leq \frac{w}{2} \\
\frac{1}{w}\left(1-\frac{x}{h_{x}}\right) & 0 \leq x \leq h_{x},|y| \leq \frac{w}{2} \\
0 & \text { elsewhere }
\end{array}\right. \\
J_{\text {Load }}(x, y)=\left\{\begin{array}{cl}
-\frac{1}{w} \frac{x+N_{1} h_{x}}{h_{x}} & -\left(N_{1}+1\right) h_{x} \leq x \leq-N_{1} h_{x},|y| \leq \frac{w}{2} \\
\frac{1}{w} \frac{x-N_{r} h_{x}}{h_{x}} & N_{r} h_{x} \leq x \leq\left(N_{r}+1\right) h_{x},|y| \leq \frac{w}{2} \\
0 & \text { elsewhere }
\end{array}\right.
\end{gathered}
$$



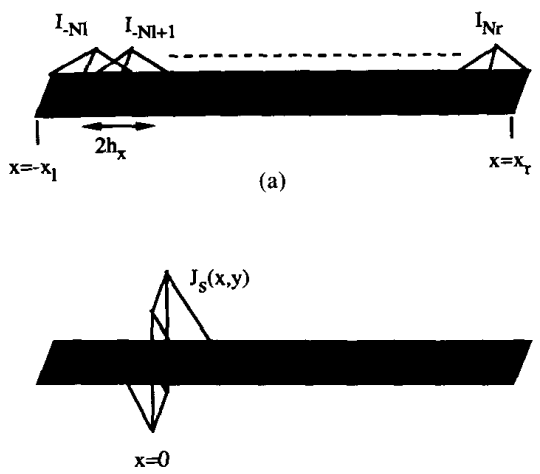

(b)

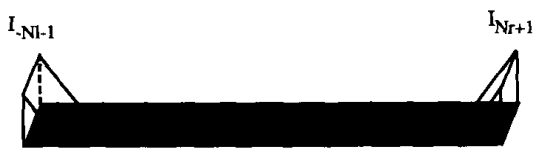

(c)

Fig. 2. Basis functions representing the current density (a) on the line, (b) at the source, and (c) at the load terminals.

and are plotted in Fig. 2(b) and (c), respectively. These basis functions have been chosen to be compatible with the rooftops (4), which have already been employed to represent the current density on the microstrip line. However, these functions are piecewise continuous while the rooftops are piecewise differentiable. Consequently, the integrals corresponding to the basis functions of the source and loads in (3) would be divergent unless we impose certain constraints that render them convergent. We will address this question next.

The problem with using piecewise continuous functions as basis functions for the current density is that, upon differentiation, they give rise to infinite, nonphysical charge density distributions. However the integrals containing these basis functions and their derivatives do become convergent once these singularities are removed. This prompts us to examine the question whether or not it is legitimate to ignore the impulse functions arising from the differentiations of the piecewise-continuous basis functions.

For the source basis function (5a), the principle of conservation of charge at the junction $x=0$, where the current-carrying probe is connected to the microstrip line, implies that the charge density cannot be singular. We can similarly argue that the divergence of the current at the load terminals must be finite. In view of this, we conclude that the singularities in the derivatives of the source and load basis functions are non-physical and should therefore be ignored wherever they appear as a result of differentiation of the current.

If we choose the basis functions given above, and ignore the singularities generated by taking the divergence of the current, we can justify the step of integration by parts in (3). Then, by transferring the derivatives in front of the convolution integrals in (3) over to the basis func- tions $J_{x m}$, as in (4), (3) can be rewritten as

$$
\begin{aligned}
& \sum_{n=-N_{1}-1}^{N_{r}+1} I_{n}\left[\left\langle J_{x m}, G_{x x}^{A} * J_{x n}\right\rangle-\frac{1}{\omega^{2}}\left\langle\frac{\partial}{\partial x} J_{x m},\right.\right. \\
& \left.\left.\cdot\left(G_{q} * \frac{\partial}{\partial x} J_{x n}\right)\right\rangle\right] \\
& =-\left\langle J_{x m}, G_{x x}^{A} * J_{s}\right\rangle+\frac{1}{\omega^{2}}\left\langle\frac{\partial}{\partial x} J_{x m},\left(G_{q} * \frac{\partial}{\partial x} J_{s}\right)\right\rangle \\
& m=-N_{1}, \cdots, N_{r}
\end{aligned}
$$

In the above equation the number of equations $\left(N_{1}+\right.$ $\left.N_{r}+1\right)$ is two less than the number of unknowns $\left(N_{1}+\right.$ $N_{r}+3$ ). However, we can supplement these missing conditions by enforcing the necessary boundary conditions at the load terminals. The procedure for doing this is detailed in the next section.

Each of the inner-product terms in (6) is a four-dimensional integral, provided that the closed-form Green's functions are used. Since the numerical integration of a four-dimensional integral is quite expensive, even though this integration is required to be carried out only over a finite range, the convolution over the Green's function and the basis function is transferred to the two basis functions involved in each term. If the basis functions have been chosen such that their convolution can be performed analytically, which is the case for the choice of the basis functions given in (4), (5a), and (5b), the inner products in (6) are reduced to double integrals over finite domains.

\section{Supplemental Equations for the Load Basis Functions}

In order to relate the coefficients of the load basis functions to those of the other basis functions, we need to impose two boundary conditions, each of which is related to the complex load impedances at the two ends. Since, at the terminations, the load impedance and the terminal current are related by the voltage difference between the line and the ground plane, these voltages can be expressed in terms of the impedances of the loads, the coefficients of the load basis functions, and the coefficients of the other basis functions used in the representation of the current on the microstrip line.

Here, we will investigate two different approaches to deriving the supplementary equations, the first of which is based on a rigorous definition of voltage in terms of field components, while the second employs a transmission line analysis using the current and voltage waves.

In the rigorous approach, the voltages at the load terminals is defined by

$$
\begin{gathered}
V\left(x=-x_{1}\right)=\int_{-d_{1}}^{0} d z E_{z 1}\left(x=-x_{1}, y=0, z\right) \\
V\left(x=x_{r}\right)=\int_{-d_{1}}^{0} d z E_{z 1}\left(x=x_{r}, y=0, z\right)
\end{gathered}
$$


where $E_{z 1}$ is the spatial domain representation of the $z$-polarized electric field in the substrate. The $z$-component of the electric field in the spectral domain can be obtained in terms of the basis functions on the microstrip line and the $z$-directed current on the probe as

$$
\tilde{E}_{1}=\tilde{G}_{z x}^{E} \tilde{J}_{x}+\tilde{G}_{z z}^{E} \tilde{J}_{z}
$$

where implies Fourier transform and superscript $E$ denotes electric field, i.e., the Green's functions in (8) are for the electric fields. It should be noted that the $z$-directed current on the probe is employed in the calculation of the $z$-polarized electric fields, although it has not been used for the calculation of the current distribution on the line (see (6)). The $x$-polarized electric field, which is used to obtain (6), is the secondary field for the $z$-directed current. At this point, it becomes obvious that there are two major disadvantages of using this rigorous approach to obtain the relationship between the load impedances and the coefficients of the basis functions. The first of these is the need to use the probe current which requires a good model for the source connection [7], while the second is the necessity to apply the computationally-expensive step of inverse Fourier transform of the $z$-polarized electric field (8) for each of the basis functions in order to obtain its spatial domain representation. In view of this, we propose a simple and computationally efficient approach based on the transmission line analysis to relate the load impedances to the surface current density on the line.

In the transmission line analysis, it is well-known that the total voltage $V(x)$ and total current $I(x)$ on the line are related by the following first-order differential equations:

$$
\begin{gathered}
\frac{d I(x)}{d x}=-Y V(x) \\
\frac{d V(x)}{d x}=-Z I(x)
\end{gathered}
$$

where $Y=j \beta / Z_{o}$ and $Z=j \beta Z_{o}$ are the series impedance and shunt admittance per unit length of the line, respectively. The characteristic impedance $Z_{o}$ and propagation constant $\beta$ of the line are calculated by using empirical formulas based on a quasi-static analysis [8]. If the derivatives in (9a) and (9b) are approximated by finite differencing and the resulting equations are related to each other for the load terminals at $x=x_{1}$ and $x=x_{r}$, the following equations are obtained (see Fig. 2(a) and 2(c)):

$$
\begin{aligned}
& \left(1+j \beta h_{x} \frac{Z_{L 1}}{Z_{0}}-\frac{\beta^{2} h_{x}^{2}}{2}\right) I_{-N_{1}-1}-I_{-N_{1}}=0 \\
& -I_{N_{r}}+\left(1+j \beta h_{x} \frac{Z_{L r}}{Z_{0}}-\frac{\beta^{2} h_{x}^{2}}{2}\right) I_{N_{r}+1}=0
\end{aligned}
$$

where $V\left(-x_{1}\right) / I\left(-x_{1}\right)=-Z_{L 1}$ and $V\left(x_{r}\right) / I\left(x_{r}\right)=Z_{L r}$ are employed. Note that (10a) and (10b) are dependent upon the finite-difference approximation; for example, here we have used central differencing for (9a) and forward differencing for (9b) to obtain (10a).
By using the (10a) and (10b) together with the equations given in (6), the current distribution on the microstrip line terminated by the complex load impedances $Z_{L 1}$ and $Z_{L r}$ is obtained. The current distribution on a transmission line fed by a unit amplitude current source at $x=$ 0 is also calculated in closed-form by using the TL approach [9], and is given by

$$
J_{x}(x)=\left\{\begin{array}{c}
-\frac{1}{2} \frac{1+e^{-j 2 k x_{r}} \Gamma_{L r}}{1-\Gamma_{L r} \Gamma_{L 1} e^{-j 2 k\left(x_{1}+x_{r}\right)}} \\
\cdot\left[e^{j k x}-\Gamma_{L 1} e^{-j k\left(x+2 x_{1}\right)}\right] \\
-x_{1} \leq x \leq 0 \\
-\frac{1}{2} \frac{1+e^{-j 2 k x_{1}} \Gamma_{L 1}}{1-\Gamma_{L r} \Gamma_{L 1} e^{-j 2 k\left(x_{1}+x_{r}\right)}} \\
\cdot\left[-e^{j k x}+\Gamma_{L r} e^{-j k\left(x-2 x_{r}\right)}\right] \\
0 \leq x \leq x_{r}
\end{array}\right.
$$

where $\Gamma_{L 1}$ and $\Gamma_{L r}$ are the voltage reflection coefficients defined on the load terminals $x=-x_{1}$ and $x=x_{r}$, respectively. The results that are obtained by the MoM and the transmission line approach (11) are presented and compared in the next section.

\section{Results and Discussions on the Current Distribution}

The following parameters have been chosen for the examples given below: the dielectric constant of the medium $\epsilon_{r 1}=4.0$; the ratio of the width of the microstrip line $w$ to the thickness $d_{1}$ of the substrate $=4.0$; the thickness of the substrate $d_{1}=8.0$ mils $(0.203 \mathrm{~mm})$; and the frequency of operation $=1.0 \mathrm{GHz}$. The current source is located at $1 \mathrm{~cm}$ away from the left edge, and has a magnitude of $2 \mathrm{~A}$.

The current distribution on a line can often be predicted intuitively for standard terminations e.g. a match, opencircuit or a short-circuit. This prompts us to use these cases as examples of our calculations. As expected, the magnitude of the current distribution becomes zero at the ends of the line while the phase shows a standing wave type of behavior and, as shown in Fig. 3(a) and (b), it switches between $0^{\circ}$ and $180^{\circ}$. Excellent agreement is observed between the current distributions calculated by the TL approach and the MoM for a microstrip line terminated at both ends by matched loads, open or short circuits, excepting in the vicinity of the resonance for the last two cases. This behavior is attributable to the difference in the resonant lengths of the line predicted by the MoM and the TL approaches. As an example of a complex termination, we have chosen a resistance of $20 \mathrm{~K}$ in parallel with a $8 \mathrm{pF}$ capacitance, which represents the typical input impedance of a TTL circuit. The current distributions for this termination have been calculated by using both the TL and the MoM approaches, and are exhibited in Fig. 4. It is observed that the magnitude of the current calculated by the TL approach is slightly different from that of the MoM, because, as mentioned above, the length 


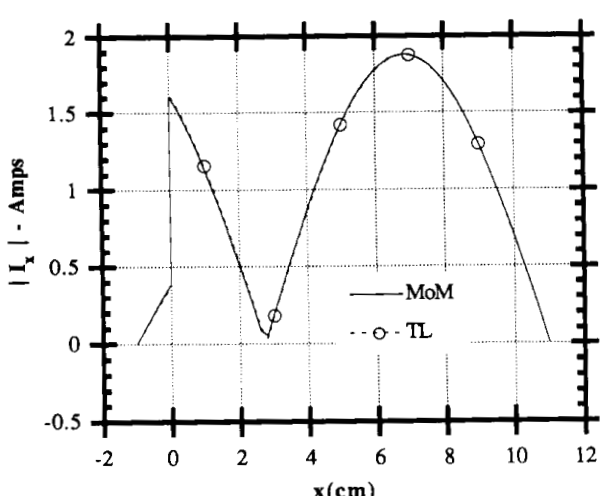

(a)

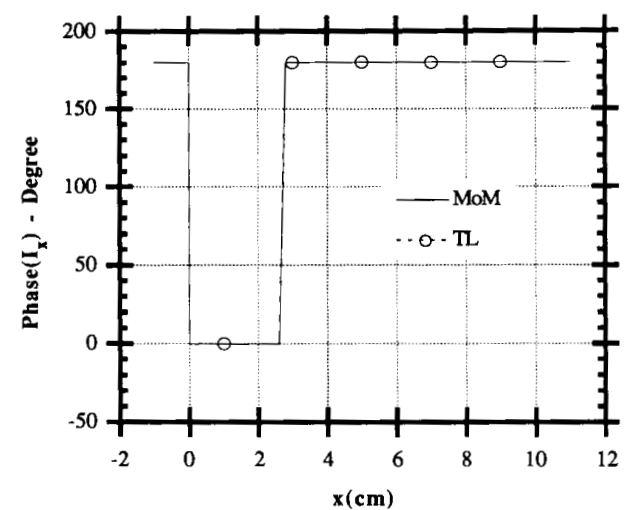

(b)

Fig. 3. Current distributions obtained by using MoM and TL approaches for open-circuited terminations; (a) magnitude, (b) phase.

of line is close to the resonant length for this load termination.

In the previous section, we stated that the propagation constant of the line is needed in (10a) and (10b) for the inclusion of the terminating loads in the formulation. This constant is calculated by using an empirical formula with approximately a 1 percent error margin. We have investigated the sensitivity of the current distribution to a change in the effective dielectric constant which, in turn, is directly related to the propagation constant. In Fig. 5 we present the plot of the current distribution on a microstrip line $12-\mathrm{cm}$ long and terminated by complex loads of $8 \mathrm{pF} / 20 \mathrm{~K} \Omega$ for two values of the effective dielectric constants, viz., $\epsilon_{r 1}=3.2$ and $\epsilon_{r l}=3.3$, which are in the vicinity of the value obtained from the empirical formula $\left(\epsilon_{r 1}=3.26\right)$. It can be observed from Fig. 5 that the current distribution remains virtually unaffected by the small variation of the effective dielectric constant.

A study of the current distributions for different lengths of the line leads us to conclude that, in general, the TL approach predicts the current distribution reasonably well, provided that the frequency of operation is not too close to the resonant frequency of the resonator represented by the truncated line. However, as we will see in the next section, the spurious radiated power is the highest at resonance.

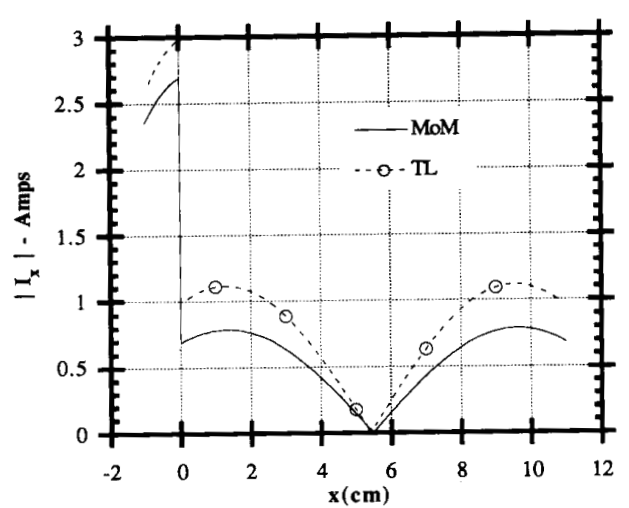

(a)

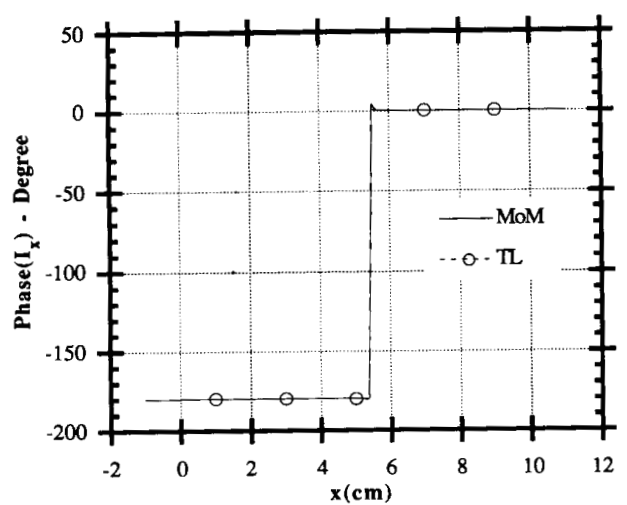

(b)

Fig. 4. Current distribution obtained by using MoM and TL approaches for a complex load termination $Z_{L}=(20 \mathrm{~K} / / 8 \mathrm{pF})$; (a) magnitude, (b) phase.

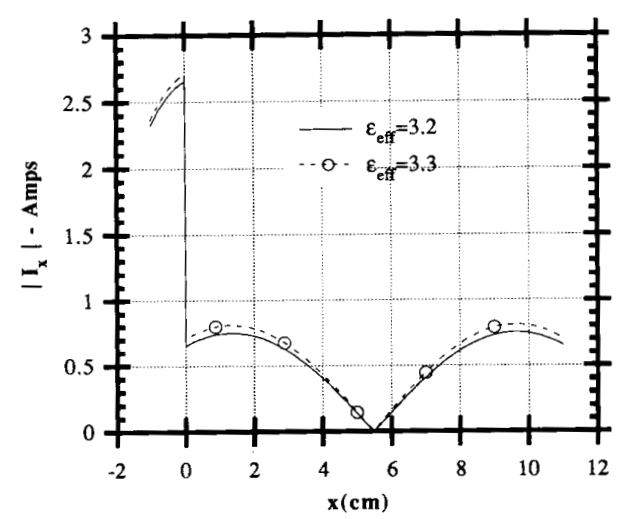

Fig. 5. Current distributions obtained by using the MoM for a complex load termination $Z_{L}=(20 \mathrm{~K} / / 8 \mathrm{pF})$ and for $\epsilon_{\mathrm{eff}}=3.2$ and 3.3 .

\section{Spurious Radiation Calculation}

Once the current distribution on the microstrip line has been derived, whether by using the TL approach or the MoM, the field distribution produced by the line currents can be readily calculated, both in the near and far-field regions, by using the field representations in terms of appropriate Green's functions. The spurious radiated power 
can then be obtained through the integration of the Poynting vector over a closed mathematical surface, e.g., a rectangular box enclosing the microstrip line.

\section{A. Formulation of the Problem}

In this section, we will calculate the spurious radiation defined as the total power crossing a plane parallel to the plane of the substrate. The total power is expressed as

$$
\begin{aligned}
P & =\frac{1}{2} \operatorname{Re} \iint_{S} d s \cdot E \times H^{*} \\
& \cong \frac{1}{2} \operatorname{Re} \iint_{S} d x d y\left(E_{x} H_{y}^{*}-E_{y} H_{x}^{*}\right)
\end{aligned}
$$

Since the calculation of the field components requires the evaluation of a convolution integral for each basis function that is used to represent the current density on the microstrip line, implementing (12) in the spatial domain becomes computationally expensive. Therefore, the field components used in (12) are transformed into the spectral domain and the total power is expressed in terms of the transform quantities as

$$
\begin{aligned}
P= & \frac{1}{2} \operatorname{Re}\left\{\frac { 1 } { 4 \pi ^ { 2 } } \int _ { - \infty } ^ { \infty } \int _ { \infty } d k _ { x } d k _ { y } \left[\hat{E}_{x}\left(k_{x}, k_{y}\right) \hat{H}_{y}^{*}\left(k_{x}, k_{y}\right)\right.\right. \\
& \left.-\hat{E}_{y}\left(k_{x}, k_{y}\right) \hat{H}_{x}^{*}\left(k_{x}, k_{y}\right)\right\}
\end{aligned}
$$

where the electric field in the spectral domain can be obtained by multiplying the spectral domain Green's functions by the Fourier transform of the current distribution on the microstrip line, which has been obtained in the previous section. Thus, the total power radiated can be written as

$$
\begin{aligned}
P= & \frac{1}{2} \operatorname{Re}\left\{\frac { 1 } { 4 \pi ^ { 2 } } \int \int _ { - \infty } ^ { \infty } d k _ { x } d k _ { y } \left[\left(\tilde{G}_{x x}^{E} \tilde{J}_{x}\right)\left(\tilde{G}_{y x}^{H} \tilde{J}_{x}\right)^{*}\right.\right. \\
& \left.\left.-\left(\tilde{G}_{y x}^{E} \tilde{J}_{x}\right)\left(\tilde{G}_{x x}^{H} \tilde{J}_{x}\right)^{*}\right]\right\}
\end{aligned}
$$

where the Green's functions are obtained by the using immittance approach [10], and given by

$$
\begin{aligned}
& \tilde{G}_{x x}^{E}=\left[\tilde{Z}^{e} \cos ^{2} \phi+\tilde{Z}^{h} \sin ^{2} \phi\right] e^{-j k_{z} 0 z} \quad \text { for } z>0 \\
& \tilde{G}_{y x}^{E}=\left[\left(\tilde{Z}^{e}-\tilde{Z}^{h}\right) \sin \phi \cos \phi\right] e^{-j k_{000}} \quad \text { for } z>0 \\
& \tilde{G}_{x x}^{H}=\left[\left(Y_{\mathrm{TE} 0} \tilde{Z}^{h}-Y_{\mathrm{TM} 0} \tilde{Z}^{e}\right) \sin \phi \cos \phi\right] e^{-j k_{x 0 z}} \\
& \quad \text { for } z>0 \\
& \tilde{G}_{y x}^{H}=\left[\left(Y_{\mathrm{TE} 0} \tilde{Z}^{h} \sin ^{2} \phi+Y_{\mathrm{TM} 0} \tilde{Z}^{h} \cos ^{2} \phi\right] e^{-j k_{z 00}}\right. \\
& \quad \text { for } z>0
\end{aligned}
$$

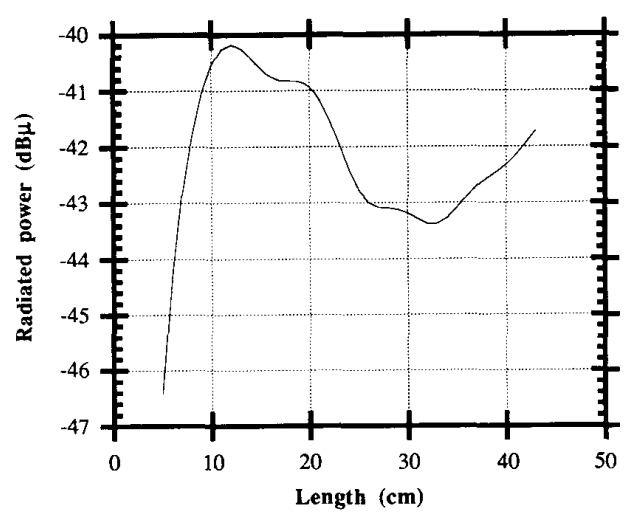

Fig. 6. Radiated power as a function of the length of the line for matched termination on both ends.

where $Y_{\mathrm{TE} 0}, Y_{\mathrm{TM} 0}$ and $Y_{\mathrm{TE} 1}, Y_{\mathrm{TM} 1}$ are wave admittances in the free space and in the dielectric medium, respectively; $\phi=\tan ^{-1}\left(k_{y} / k_{x}\right)$; and,

$$
\begin{aligned}
\tilde{Z}^{e} & =\frac{-1}{Y_{\mathrm{TM} 0}-j Y_{\mathrm{TM} 1} \cot \left(k_{z 1} d_{1}\right)} ; \\
\tilde{Z}^{h} & =\frac{-1}{Y_{\mathrm{TE} 0}-j Y_{\mathrm{TE} 1} \cot \left(k_{z 1} d_{1}\right)}
\end{aligned}
$$

Since the value of $z$ is greater than zero, the Green's functions (15a)-(15d) become decaying functions for a bulk of the spectral components, and this leads to the rapid convergence of the double integral (14).

\section{B. Results and Discussions}

In this part of the study, the length of the line is considered to be the independent variable while the spurious radiated power is viewed as the dependent one. The dielectric constant of the medium is $\epsilon_{r 1}=4.0$, the width of the line $w$ to the thickness of the substrate $d_{1}$ ratio is 4.0 , and the thickness of the substrate is $d_{1}=8.0$ mils $(.203$ $\mathrm{mm}$ ). The current source is again located $1.0 \mathrm{~cm}$ from the left edge of the microstrip line; however, its amplitude is normalized to $1 \mathrm{~mA}$ for the calculation of spurious radiated power.

The spurious power, as defined by Equation (14), is calculated for a microstrip line terminated by matched loads, open circuits and short circuits at both terminals and, a matched load on the left and a complex load of 8 $\mathrm{pF} / / 20 \mathrm{~K} \Omega$ on the right terminal. The results are given in Figs. 6, 7, 8, and 9, respectively. The radiation from a microstrip line terminated by a pair of matched loads is very small (see Fig. 6) as compared to those terminated by other loads. The highest radiation occurs for the opencircuited and the short-circuited transmission lines of resonant lengths. The radiated power has a sharp peak around the resonance length of the line, and it becomes essentially negligible for off-resonance lengths. For the combination of matched and complex load terminations, Fig. 9 , the total radiated power is slightly larger than that of matched load termination case shown in Fig. 6. 


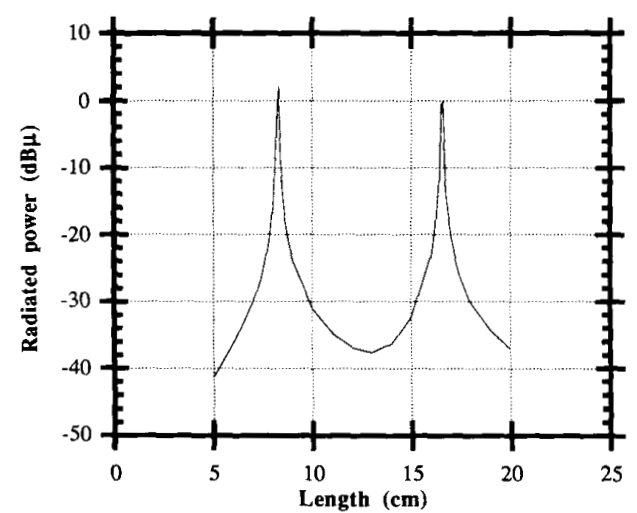

Fig. 7. Radiated power as a function of the length of the line for opencircuit termination at both ends.

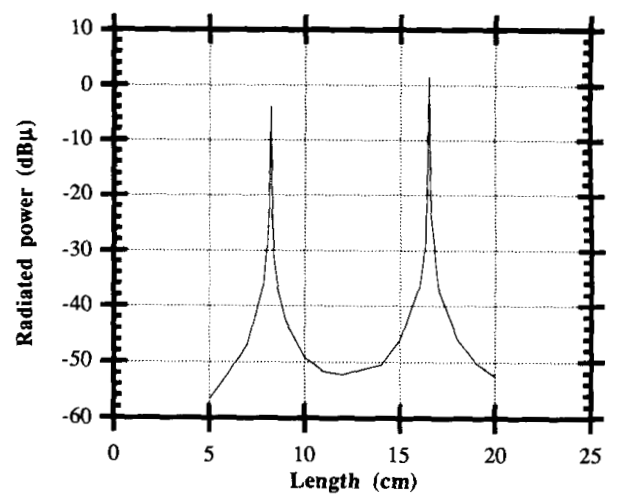

Fig. 8. Radiated power as a function of the length of the line for shortcircuit termination at both ends.

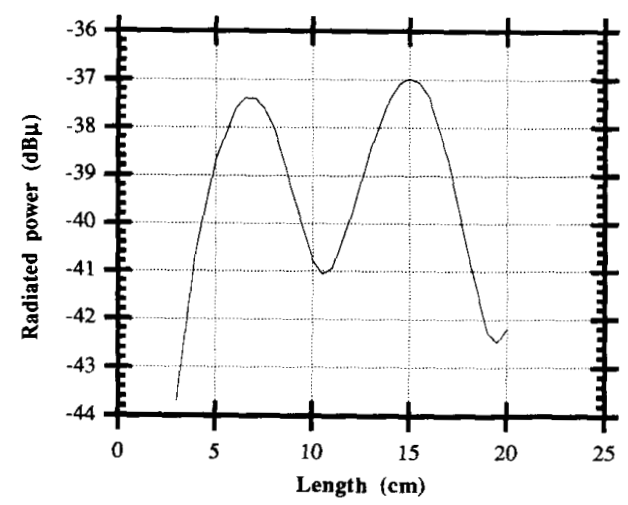

Fig. 9. Radiated power as a function of the length of the line for a matched load and a complex load $Z_{L}=(20 \mathrm{~K} / / 8 \mathrm{pF})$ terminations.

\section{Conclusions}

The current distribution on a microstrip line, which is fed by a current source at an arbitrary location and terminated by complex loads at both ends, has been computed by using the closed-form representations of the spatial domain Green's functions.

It has been found that the use of closed-form spatial domain Green's functions in the context of the method of moments formulation reduces the computation time significantly as compared to the conventional formulation in the spectral domain. For instance, in a numerical experiment with 40 roof-top basis functions, the computation time for the current distribution is on the order of 50-60 CPU sec on the DECstation 5000/200 system when the closed-form Green's functions in the format given in [4] are used, whereas it takes on the order of $10 \mathrm{CPU}$ mins. on Cray/YMP for the same calculation using the spectral domain approach. The method described is quite general and is useful for arbitrary geometrical disposition of the microstrip etches, e.g., arbitrary bends, and not just straight sections.

The investigation of the radiation leakage from a microstrip line terminated by complex loads has shown that the highest radiation occurs when the length of the line is near resonance, and the terminations are either open or short circuits. It is also observed that a matched load termination at one of the terminals of the microstrip interconnect reduces the radiation leakage significantly, as compared to the radiation levels for other terminations that can cause resonances to occur.

\section{ACKNOWLEDGMENT}

This work was supported in part by the Joint Services Electronics Program under Grant N00014-90-J-1270. Support from the National Center for Supercomputing Applications at the University of Illinois for computer time on the Cray/YMP supercomputer is also acknowledged.

\section{REFERENCES}

[1] R. F. Harrington, Field Computation by Moment Method. New York, MacMillan, 1968

[2] Y. L. Chow, J. J. Yang, D. H. Fang and G. E. Howard, "Closedform spatial Green's function for the thick substrate," IEEE Trans. Microwave Theory Tech., vol. 39, pp. 588-592, Mar. 1991

[3] Y. L. Chow and J. J. Yang, "Modeling of electromagnetic fields in layered media by the simulated image technique," Directions in Electromagnetic Wave Modeling, H. L. Bertoni, and L. B. Felsen, Eds., New York: Plenum, 1991.

[4] M. 1. Aksun and R. Mittra, "Deviation of closed-form Green's functions for a general microstrip geometry," accepted for publication in IEEE Trans. Microwave Theory Tech.

[5] _- "Choices of basis and testing functions for the method of moments in electromagnetic problems," submitted for publication.

[6] R. A. York, R. C. Compton, and B. J. Rubin," Experimental verification of the 2-D rooftop approach for modeling microstrip path antennas," IEEE Trans. Antennas Propagat., vol. 39, pp. 690-694, May 1991.

[7] J. T. Aberle and D. M. Pozar, "Analysis of infinite arrays of probefed rectangular microstrip patches using a rigorous feed model," Proc. Inst. Elec. Eng., vol. 136, pp. 110-119, Apr. 1989.

[8] H. Sobol, "Application of integrated circuit technology to microwave frequencies," Proc. IEEE, vol. 59, pp. 1200-1211, Aug. 1971.

[9] C. T. Tai, Dyadic Green's Functions in Electromagnetic Theory. San Francisco: International Textbook 1971.

[10] T. Itoh, "Spectral domain immittance approach for dispersion characteristics of generalized printed transmission lines," IEEE Trans. Microwave Theory Tech. vol. MTT-28, pp. 733-736, July 1980.

M. Irsadi Aksun, for a photograph and biography, see this issue, p. 2062.

Raj Mittra (S'54-M'57-SM'69-F'71), for a photograph and biography, see this issue, p. 2062. 